\title{
Modelling of diffusion in porous structures
}

\author{
E. du Plessis \& S. Woudberg \\ Applied Mathematics, Department of Mathematical Sciences, \\ Stellenbosch University, South Africa
}

\begin{abstract}
An existing pore-scale model is used to predict the effective diffusivity of staggered two-dimensional rectangular unconsolidated arrays through the use of a Representative Unit Cell concept. A tri-diagonal matrix algorithm is used to solve the diffusive flux field and to compute the effective diffusion coefficient for concentration gradients of staggered arrays. The numerical results and analytical model are compared critically with theoretical and numerical studies, as well as experimental data reported in literature. The good correlations obtained for the effective diffusivity provide confidence in both the computational and analytical work.
\end{abstract}

Keywords: diffusion, porous media, pore-scale, effective diffusion coefficient, modelling, microstructure, fibres.

\section{Introduction}

The study of molecular diffusion forms an important cornerstone in the analysis of more involved multiphase processes, such as dispersion and combustion in porous media, which form part of many processes in the coal industry, e.g. the recovery of methane from coal beds (Kim et al. [1]) and similar industries. Natural substances, such as coal, vary considerably in structural morphology. The microstructure is seldom simple and most often present a mixture of different types of porous environments. The flow processes thus often consist of a mixture of convective flow in macropores and molecular diffusion in micropores. Following up on considerable success with a pore-scale model on the modelling and subsequent prediction of drag during convection in porous media, our next goal is the modelling of diffusion making use of the same geometrical and modelling practices. To this end, descriptions of diffusion in two-dimensional rectangular arrays of non-staggered 
and of fully staggered solid rectangles are needed to formalize the theory and to compare it with published experimental, computational and theoretical models.

\section{Diffusion equation}

Fick's second law of diffusion or simply the diffusion equation is given by

$$
\frac{\partial \rho_{A}}{\partial t}+\nabla \cdot D \nabla \rho_{A}=0,
$$

where $D$ is the mass diffusivity or diffusion coefficient for component A diffusing through component $\mathrm{B}$ and $\rho_{A}$ is the mass concentration of species $\mathrm{A}$. The diffusivity $D$ is a property of a specific system, dependent upon the system's temperature, pressure and composition (Welty et al. [2]). Eqn. (1) applies to a stationary, incompressible fluid without chemical production. For a steady incompressible fluid, constant diffusion coefficient, no chemical production and no fluid motion the Laplace equation for mass transport is obtained (Welty et al. [2]), i.e.

$$
\nabla^{2} \rho_{A}=0
$$

\section{Effective diffusion coefficients of porous media}

Equation (2) is a point equation which governs the transport of mass of the chemical species considered. When regarding diffusion in porous media, eqn (2) needs to be solved at every point within the porous medium which leads to enhanced mathematical complexity. The characterization of diffusion in porous media therefore relies on the use of macroscopic dependent variables (Sáez et al. [3]). Eqn (2) thus needs to be volume averaged over a representative portion of the porous domain according to the volume averaging theory (e.g. Whitaker [4]). Boundary conditions also need to be imposed together with conditions at the fluidsolid interfaces (Sáez et al. [3]). For the present work no mass transfer between the phases will be considered. Volume averaging of eqn (1) leads to the following macroscopic diffusion equation:

$$
\frac{\partial\left\langle\rho_{A}\right\rangle_{f}}{\partial t}=D_{e f f}: \nabla \nabla\left\langle\rho_{A}\right\rangle_{f},
$$

where $D_{\text {eff }}$ is the effective diffusion coefficient which is only a function of the geometric structure of the porous medium at the pore level (Sáez et al. [3]) and may thus be expressed as a function of the porosity alone, i.e.

$$
\frac{D_{e f f}}{D}=f(\epsilon) \text {. }
$$

In the next section a geometric pore-scale model will be introduced which will be used to predict the effective diffusivity analytically. 


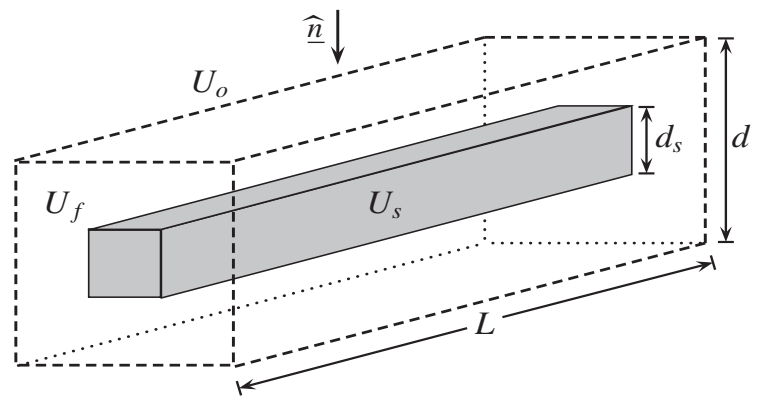

Figure 1: RUC model for fibre beds.

\section{Geometric pore-scale model for fibre beds}

The geometric pore-scale model for fibre beds was originally introduced by $\mathrm{Du}$ Plessis [5]. It is based on a rectangular Representative Unit Cell, abbreviated RUC, which is defined as the smallest rectangular control volume, $U_{o}$, into which the average geometrical properties of the porous medium may be embedded. The RUC model for fibre beds is shown in Fig. 1.

Only cross-flow - flow perpendicular to the prism axis - will be considered, as indicated by the streamwise direction $\underline{\widehat{n}}$ in Fig. 1. Cross-flow through prismatic porous media can therefore be approximated as two-dimensional flow. Two arrays are considered, namely a fully staggered array in which maximum staggering occurs in the streamwise direction and a non-staggered array in which no staggering occurs in the streamwise direction. The fluid filled volume within the RUC is denoted by $U_{f}$ and $U_{s}$ denotes the volume of the solid phase. The RUC model is assumed to be homogeneous and isotropic with respect to the average geometric properties of the fibre bed. The porosity $\epsilon$ of the RUC model is defined as

$$
\epsilon=\frac{U_{f}}{U_{o}}
$$

\subsection{Volume partitioning}

The total fluid filled volume $U_{f}$ within the RUC may be expressed as

$$
U_{f}=U_{\|}+U_{\perp}+U_{g}+U_{t},
$$

where the streamwise volume $U_{\|}$is the total fluid volume parallel to the streamwise direction, the transverse volume $U_{\perp}$ is the total fluid volume perpendicular to the streamwise direction, in the stagnant volume, $U_{g}$, the fluid remains stationary and in the transverse fluid volume, $U_{t}$, no wall friction occurs due to the absence of adjacent solid surfaces. From eqn (5) and quantification of the respective fluid volumes, the following relationship between the linear dimensions of the RUC 
model for fibre beds is obtained in terms of the porosity (Du Plessis [5]):

$$
d_{s}=d \sqrt{1-\epsilon} \text {. }
$$

Diedericks and Du Plessis [6] defined the geometrical tortuosity as the ratio of the streamwise displacement $d$ to the total path length $d_{e}$ of the fluid traversing through the constant cross-sectional area $A_{p_{\|}}$in the RUC. The tortuosity may also be expressed as a volumetric ratio, but with stagnant volumes excluded, yielding [7]

$$
\chi=\frac{d_{e}}{d}=\frac{U_{\|}+U_{t}+\xi U_{\perp}}{A_{p_{\|}} d}=\frac{U_{\|}+U_{t}+\xi U_{\perp}}{U_{\|}+U_{t}},
$$

where the coefficient $\xi$ was introduced to account for the reduction in the tortuosity due to the splitting of the streamtube into two equal but directionally opposite transverse parts in a fully staggered array. It thus follows that $\xi=1 / 2$ for the fully staggered array and $\xi=0$ for the non-staggered array. Quantification of the tortuosity leads to the following expression for the fully staggered array

$$
\chi=1+\frac{1}{2} \sqrt{1-e}
$$

and for the non-staggered array $\chi=1$. In order to account for the effect of stagnant regions a geometric factor $\psi$ was introduced and defined as (Lloyd et al. [7])

$$
\psi \equiv \frac{U_{f}}{U_{\|}+U_{t}}=\frac{U_{f}}{A_{p_{\|}} d}=\frac{U_{\|}+U_{t}+U_{\perp}+U_{g}}{U_{\|}+U_{t}} .
$$

The geometric factor expressed as a function of the porosity alone yields

$$
\psi=\frac{\epsilon}{1+\sqrt{1-e}} .
$$

\subsection{Effective diffusivity predicted by the RUC model}

According to Kim et al. [1] it is common for isotropic processes to express the effective diffusivity $D_{\text {eff }}$ over the diffusivity $D$ as

$$
\frac{D_{e f f}}{D}=\frac{\epsilon}{\chi}
$$

Making use of eqn (9) for the tortuosity obtained by the RUC model, yields

$$
\frac{D_{e f f}}{D}=\frac{\epsilon}{1+\frac{1}{2} \sqrt{1-e}}
$$

for the fully staggered array. An alternative expression for the effective diffusion coefficient can be obtained by using the geometric factor supplied by the RUC 
model, which takes into account stagnant regions, instead of the tortuosity, i.e.

$$
\frac{D_{e f f}}{D}=1+\sqrt{1-e} .
$$

Equation (14) applies to both the staggered and non-staggered arrays.

Weissberg [8] made use of a variational approach which they applied to a bed of spheres to obtain the following expression for the effective diffusivity:

$$
\frac{D_{e f f}}{D}=\epsilon\left(1-\frac{1}{2} \ln \epsilon\right)^{-1}
$$

Maxwell (Kim et al. [1]) analyzed a dilute suspension of spheres analytically and obtained the following expression for the effective diffusivity:

$$
\frac{D_{e f f}}{D}=\epsilon\left[1+\frac{1}{2}(1-\epsilon)\right]^{-1} \text {. }
$$

The micropore-macropore model of Wakao and Smith [9] is given by

$$
\frac{D_{e f f}}{D}=\epsilon^{2}
$$

Based on their experimental data Kim et al. [1] proposed the following empirical equation for the effective diffusivity:

$$
\frac{D_{e f f}}{D}=\epsilon^{1.4} .
$$

\section{Series-parallel formulae}

A popular concept to model diffusion in arbitrary composites is to mix two formulae that applies to composites made up of laminates of different diffusivities, namely the series and the parallel formula (Crank [10]). The assumptions for these models are that each laminate has uniform diffusive properties and the diffusive transport is uni-directional. It will be assumed that the solids are impenetrable, i.e. $D_{s}=0$ (Crank [10]).

\subsection{Series-parallel (SP) model}

In the SP model (Crank [10]) the composite is split into thin cross-stream strips, after which an effective diffusion coefficient is calculated for each strip using the parallel formula and, finally, by summing the strips in series an effective diffusion coefficient is obtained for the composite as a whole. Thus, in the SP-model the parallel formula is first applied, followed by the series formula. 


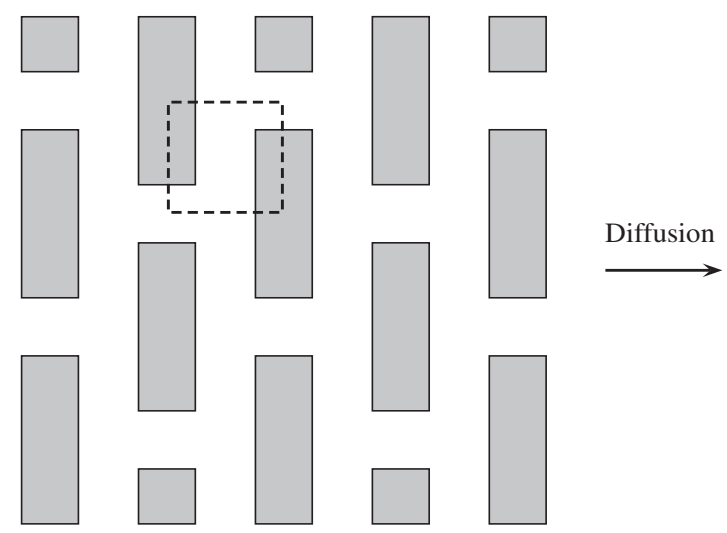

Figure 2: Overlapping fully streamwisely staggered array.

\subsection{Parallel-series (PS) model}

In the PS model (Crank [10]) the composite is split into thin strips parallel to the streamwise direction, after which an effective diffusion coefficient is calculated for each strip using the series formula and, finally, by summing the strips in parallel an effective diffusion coefficient is obtained for the composite as a whole. Thus, in the PS-model the series formula is first applied, followed by the parallel formula.

\section{Effective diffusion coefficients of ordered arrays}

For the present work only the overlapping fully streamwisely staggered array will be discussed. A regular array, a non-overlapping fully staggered array and an overlapping fully transversally staggered array have also been studied but will not be included in this work.

In a regular array no staggering and no overlap of the solids occur in any of the two principle directions. In the non-overlapping fully staggered array the solid rectangles are staggered in both principle directions but with no overlap of the solids. In the overlapping fully streamwisely staggered array staggering and overlapping of the solids occur only in the streamwise direction. A schematic representation of an overlapping fully streamwisely staggered array is shown in Fig. 2. The unit cell is indicated by the dashed lines and also shown in Fig. 3.

Both the SP and the PS models were applied to the unit cell. The results of the overlapping and non-overlapping streamwisely staggered arrays are presented in Table 1. 


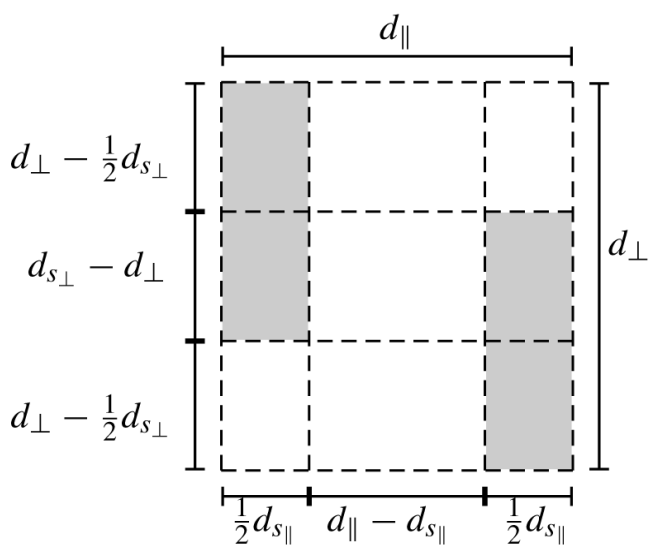

Figure 3: Unit cell of streamwise fully staggered array with overlaps.

Table 1: Diffusion coefficients based on the SP- and PS-models.

\begin{tabular}{|r|c|}
\hline $\begin{array}{r}\text { Non-overlapping fully } \\
\text { staggered array: SP model: }\end{array}$ & $\frac{D_{S P}}{D}=\left[1+\frac{d_{S_{\|}} d_{S_{\perp}}}{d_{\|}\left(2 d_{\perp}-d_{S_{\perp}}\right)}\right]^{-1}$ \\
PS model: & $\frac{D_{P S}}{D}=\frac{d_{\perp}-d_{S_{\perp}}}{d_{\perp}}$ \\
\hline $\begin{array}{r}\text { Overlapping fully streamwisely } \\
\text { staggered array: SP model: }\end{array}$ & $\frac{D_{S P}}{D}=\left[1+\frac{d_{S_{\|}} d_{S_{\perp}}}{d_{\|}\left(2 d_{\perp}-d_{S_{\perp}}\right)}\right]^{-1}$ \\
PS model: & $\frac{D_{P S}}{D}=0$ \\
\hline
\end{tabular}

\section{Weighted average of the SP and PS models}

The following weighted average is suggested by Bell and Crank [11] to predict the effective diffusivity:

$$
D_{\text {eff }}=\theta D_{S P}+(1-\theta) D_{P S},
$$

where $D_{S P}$ and $D_{P S}$ are the estimates produced by the SP and PS models and

$$
\theta=0.56-0.5\left(\frac{1}{2} d_{s_{\|}}\right)+0.4\left(\frac{1}{2}\left(d_{\perp}-d_{s_{\perp}}\right)\right) .
$$




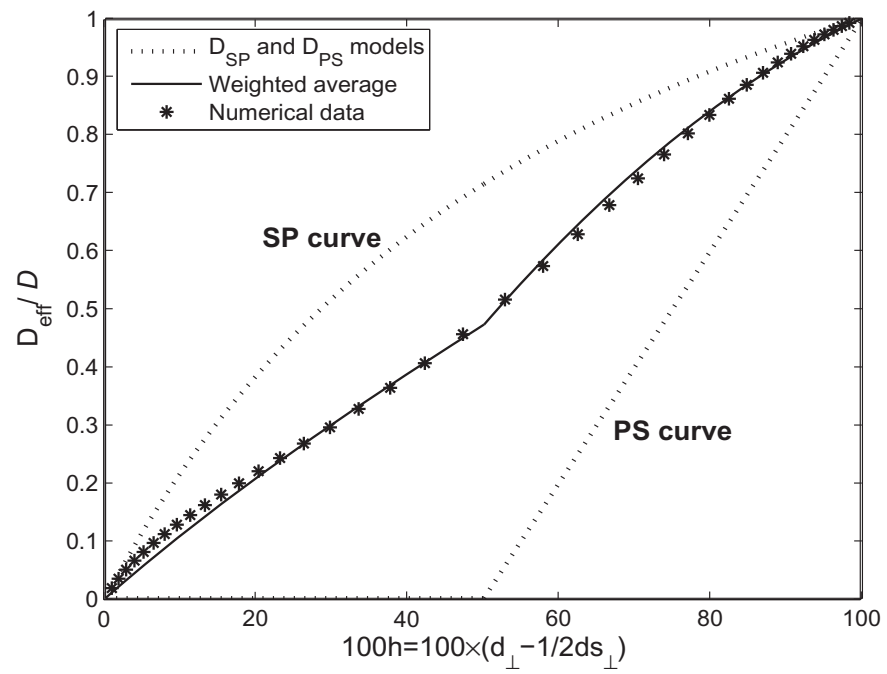

Figure 4: Effective diffusion coefficient as a function of the transverse pore width $h$ for $\sigma=0.2$.

For the present analysis let the streamwise solid dimension be denoted by $\sigma=$ $\frac{1}{2} d_{S_{\|}}$and the transverse pore width be denoted by $h=\frac{1}{2}\left(d_{\perp}-d_{s_{\perp}}\right)$.

\section{Numerical computations}

A tri-diagonal matrix algorithm was used to solve the diffusive flux field and to compute the concentration gradients from the discritized form of eqn (2). The concentration values were used to calculate the effective diffusion coefficient. The ratio of $D_{\text {eff }} / D$ was calculated as the ratio of the sum of the fluxes of all the cells within the porous matrix over the ratio of the total flux when no solids are present.

\subsection{Fully staggered array of rectangles}

Fig. 4 shows the effective diffusion coefficient as a function of the transverse pore width $h$ for $\sigma=0.2$. In RUC notation it follows that for this specific case $d_{\|}=d_{\perp}$ and $\frac{1}{2} d_{s_{\|}}=0.2$, whilst $\frac{1}{2}\left(d_{\perp}-d_{S_{\perp}}\right)$ is allowed to vary. For $0 \leq h \leq 0.5$ the SP and PS models for the overlapping fully streamwisely staggered array were used and for $0.5 \leq h \leq 1$ the SP and PS models for the non-overlapping fully staggered array were used. The predictions by both the SP and PS models are shown in Fig. 4 together with the weighted average of the two models. The agreement between the weighted average model and the numerical data is satisfactory. Fig. 4 is similar to Fig. 9(a) of Bell and Crank [11]. 


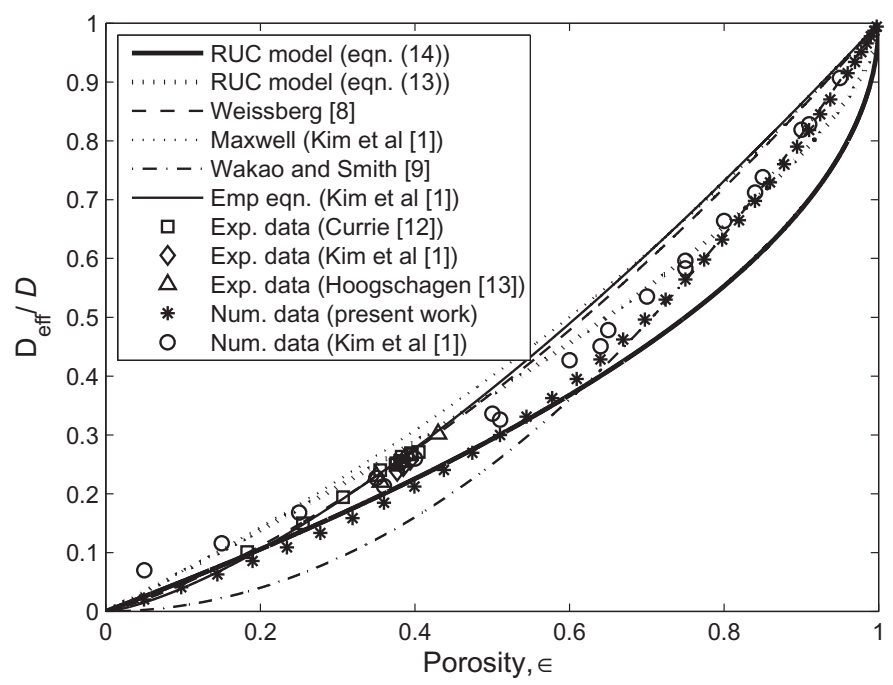

Figure 5: Effective diffusion coefficient as a function of porosity for a fully staggered array of squares.

\subsection{Fully staggered array of squares}

The effective diffusion coefficient for the fully staggered isotropic array was computed by setting $2 d_{\|}=d_{\perp}$, because if a square cell with solid squares is used, then the effective diffusion coefficient cannot be computed for porosities less than 0.5 because the solid squares will begin to overlap. In the way Bell and Crank [11] formulated their cells it is most probable that they encountered this restriction for further analysis. The effective diffusion coefficients predicted by the SP and PS models for square arrays can be obtained from table 1 as a function of the porosity by setting $d_{\|}=d_{\perp}=d$ and $d_{s_{\|}}=d_{s_{\perp}}=d_{s}$.

Fig. 5 compares the numerical data to the predictions for the effective diffusivity obtained by the RUC model and with experimental and numerical data as well as several analytical models from literature.

The model of Wakao and Smith [9] underpredicts the diffusivities obtained from the experimental data whilst both RUC models are in good agreement with the experimental data as well as all the other analytical models. The experimental data of Currie [12] and Hoogschagen [13] were obtained from beds of spheres. The RUC model involving the tortuosity proves to be more accurate at porosities greater than 0.8 than the RUC model based on the geometric factor. Nevertheless, both RUC models as well as the numerical results obtained within this work are in good agreement with the experimental and numerical data from literature as well as with the other analytical models from literature. Kim et al. [1] states that simple two-dimensional models can be used to predict the transport properties of isotropic systems which is confirmed by the present work. 


\section{Conclusions}

A pore-scale model of simple rectangular geometry is used to predict the effective diffusion coefficient for isotropic systems. The present results show that the effective diffusivity can be accurately predicted in terms of only the porosity and the particle geometry. Numerical computations were performed to compute the effective diffusion coefficient for staggered arrays of solid rectangles. Both the analytical RUC model and the numerical computations are in good agreement with data and predictive models from literature. The successful interim results pave the way for a more advanced study towards analysis of coupled convectivediffusive processes in double porosity media which is important in the study of coal processing.

\section{References}

[1] Kim, J., Ochoa, J.A. \& Whitaker, S., Diffusion in Anisotropic Porous Media. Transport in Porous Media, 2, pp. 327-356, 1987.

[2] Welty, J.R., Wicks, C.E. \& Wilson, R.E., Fundamentals of Momentum, Heat and Mass Transfer. John Wiley and Sons, 1969.

[3] Sáez, A.E., Perfetti, J.C. \& Rusinek, I., Prediction of Effective Diffusivities in Porous Media using Spatially Periodic Models. Transport in Porous Media, 6, pp. 143-157, 1991.

[4] Whitaker, S., The Method of Volume Averaging. Kluwer Academic Publishers, 1999.

[5] Du Plessis, J.P., Saturated crossflow through a two-dimensional porous medium. Advanced Water Resources, 14(3), pp. 131-137, 1991.

[6] Diedericks, G.P.J. \& Du Plessis, J.P., On tortuosity and areosity tensors for porous media. Transport in Porous Media, 20, pp. 265-279, 1995.

[7] Lloyd, C.A., Du Plessis, J.P. \& Halvorsen, B.M., On Closure Modelling of Volume Averaged Equations for Flow Through Two-Dimensional Arrays of Squares. Proceedings of the Fifth International Conference on Advances in Fluid Mechanics, March 2004, Lisbon, Portugal, pp. 85-93, 2004.

[8] Weissberg, H.L., Effective Diffusion Coefficient in Porous Media. Journal of Applied Physics, 34(9), pp. 2636-2639, 1963.

[9] Wakao, N. \& Smith, J.M., Diffusion in catalyst pellets. Chemical Engineering Science, 17, pp. 825-834, 1962.

[10] Crank, J., The Mathematics of Diffusion. Clarendon Press, Oxford, 1975.

[11] Bell, G.E. \& Crank, J., Influence of imbedded particles on steady-state diffusion. J Chem Soc, Farabay Trans 2, 70, pp. 1259-1273, 1974.

[12] Currie, J.A., Gaseous diffusion in porous media part 1. - a non-steady state method. British Journal of Applied Physics, 11, pp. 314-324, 1960.

[13] Hoogschagen, J., Diffusion in Porous Catalysts and Adsorbents. Industrial and Engineering Chemistry, 47, pp. 906-913, 1955. 